\title{
XIV. Note on capillarity constants of crystal faces
}

\section{Harold Hilton}

To cite this article: Harold Hilton (1902) XIV. Note on capillarity constants of crystal faces, Philosophical Magazine Series 6, 3:13, 144-148, DOI: 10.1080/14786440209462747

To link to this article: http://dx.doi.org/10.1080/14786440209462747

曲 Published online: 09 Jun 2010.

Submit your article to this journal $\pi$

Џll Article views: 2

Q View related articles ¿

Citing articles: 1 View citing articles $\square$ 
of sodium could be shown in the same way, though the one experiment that I tried with it gave negative results.

I expect in the near future to investigate all of the questions which $I$ have touched upon in the latter part of this paper, for it appears to me that, with proper coaxing, sodium vapour may furnish us with much information regarding molecular dynamics. The results obtained up to the present time are only a small heginning, but appear to indicate that in sodium vapour we have a medium which approaches more nearly to the ideal absorbing medium, with but a single natural period of vibration, thim any substance heretofore investigated.

Of course I am speaking here only with reference to the natural vibration which appears to influence the dispersion. Strictly speaking there are two natural periods of course, which infuence the velocity of the light in the medium, but when the medium is very dense the condition certainly approaches very nearly to that of a single period medium. As 1 have said before, the fluted absorption-bands are without influence on the dispersion, at least their influence is too slight to be detected by the methods that have been emplojed thus far.

XIV. Note on Capillarity Constants of Crystal Faces. By Harold Hilín *.

N page 520 of the 34 th volume of the Zeitschr. f. Kryst. u. Min. G. Wulff gives a very interesting theorem; he says:- "Nelmen wir an, dass wir ein Polyëder mit gegebenen Flächenrichtungen, welche durch die Normalen $n_{1}, n_{2}, n_{3} \ldots$ bestimmt werden, gefunden haben, welches der Bedingung des Minimums der Oberflächenenergie bei gegebenem Volumen Genüge leistet. Es ist einleuchtend, dass alle Polyëder welche dem letzteren ähnlich sind und nur durch ihre Volumen sich von einander unterscheiden, ebenfalls dieser Bedingung Genüge leisten werden. Alle dieser Polyëder werden einen Krystall in verschiedenen Wachstumsstadien vorstellen, wobei es klar ist, dass der Wachstumsanfang mit dem letzten Centrum der Aehnlichkeit der ganzen erhaltenen Polyëderreihe zusammenfallen muss. Auf solche Weise kann man die Oberfläche eines dieser Polyëder folgendermassen ausdrucken : $p\left(n_{1}{ }^{2}+n_{2}{ }^{2}+n_{3}{ }^{3}+\ldots\right)$, wo $p$ eine Constant ist, und die Oberffïchenenergie $\mathbf{E}$ bei den Capillaritätsconstanten $k_{1}, k_{2}, k_{3}, \ldots$ auf den verschiedenem Flächen

$$
\mathrm{E}=p\left(k_{1} n_{1}{ }^{2}+k_{2} n_{2}{ }^{2}+k_{3} n_{3}^{2}+\ldots\right)
$$

* Communicated by the Author. 
sein wird. Das Volumen dieses Polyëders kann in folgender Form geschrieben werden :

$$
\mathrm{V}=q\left(n_{1}{ }^{3}+n_{z}{ }^{3}+n_{3}{ }^{3}+\ldots\right)
$$

wobei $q$ eine (Constante ist.

" Da das Polyëder ein Minimum der Oberflächenenergie bei constanten Volumen besitzen muss, so müssen folgende Bedingungen erfüllt werden :

$$
\begin{aligned}
& d \mathrm{E}=2 p\left(k_{1} n_{1} d n_{1}+k_{2} n_{2} d n_{2}+k_{3} n_{3} d n_{3} \ldots\right)=0 ; \\
& d \mathrm{~V}=3 q\left(n_{1}^{2} d n_{1}+n_{2}^{2} d n_{2}+n_{3}^{2} d n_{3}+\ldots\right)=0,
\end{aligned}
$$

dieses ist aber nur dann möglich, wenn

$$
k_{1}: k_{2}: k_{3} \ldots=n_{1}: n_{2}: n_{3}: \ldots "
$$

'l'his proof, however, is faulty; for in writing down the expressions for $\mathrm{E}$ and $\mathrm{V}$ - which should be $\left(k_{1} p_{1} n_{1}{ }^{2}+k_{2} p_{2} n_{2}{ }^{2}+\ldots\right)$ and $\frac{1}{3}\left(p_{1} n_{1}{ }^{y}+p_{2} n_{2}{ }^{3}+\ldots\right)$ respectively, where $p_{1}, p_{2}, \ldots$ are constants : this, however, does not affect his argument,he has assumed the ratios $n_{1}: n_{2}: n_{3} \ldots$ constant, and therefore in the expressions for $d \mathrm{E}$ and $d \mathrm{~V}, d n_{1}: d n_{2}: d n_{3} \ldots$ will be constant; but in deducing $k_{1}: k_{2}: k_{3} \ldots=n_{1}: n_{2}: n_{3} \ldots$ from $d \mathrm{E}=d \mathrm{~V}=0$ he tacitly assumes $d n_{1}, d n_{2}, d n_{3} \ldots$ quite independent. His result, however, is correct, as the following proof shows:-

Suppose $s_{1}, s_{2}, s_{3} \ldots$ the areas of the faces $\sigma_{1}, \sigma_{2}, \sigma_{3} \ldots$ perpendicular to the normals through any point whose lengths are $n_{1}, n_{2}, n_{3} \ldots$ respectively. We have then

$$
\mathrm{V}=1 \mathrm{~s} \Sigma s \text { and } \therefore d \mathrm{~V}=\frac{1}{3} \mathrm{\Sigma}(n d s+s d n) \text {. }
$$

Now if a polyhedron undergoes a small deformation, the normals to its faces remaining fixed in direction, we have $d \mathrm{~V}=\Sigma s d n$; for suppose the polyhedron to be immersed in a weightless incompressible fluid which is contained in a cylinder of eross-section A, closed at the top with a movable piston of weight $W$. Then the pressure at any point of the liquid is $\frac{\mathbb{H}}{\mathrm{A}}$.

If now the polyhedron undergoes a small deformation of the kind stated above, the work done by any surface lies between

$$
\frac{\mathrm{W}}{\mathrm{A}}(s+d s) d n \text { and } \frac{\mathrm{W}}{\mathrm{A}} s d n \text {, and } \therefore=\frac{\mathrm{W}}{\overline{\mathrm{A}}} s d n,
$$

neglecting smill quantities of the second order. If now the polyhedron's volume increases by a quantity $d \mathrm{~V}$, the piston is Plil. Mag. S. 6. Vol. 3. No. 13. Jan. 1902. 
forced through a height $\frac{d \mathrm{~V}}{\mathrm{~A}}$; and therefore the work done by the surfaces of the polyhedron is $\mathrm{W} \frac{d \mathbf{V}}{\mathbf{A}}$;

Hence

$$
\text { and } \therefore \mathrm{W}_{\mathrm{A}}^{d \mathrm{~V}}=\Sigma \frac{\mathrm{W}}{\mathrm{A}} s d n \text {, and } \therefore d \mathrm{~V}=\Sigma s d u \text {. }
$$

$$
d V=1\{\Sigma(n d s)+d V\} \text { and } \therefore \Sigma_{n d s=2 d V} \text {. }
$$

Now we have relations between $s_{1}, s_{2}, s_{3} \ldots$ due to the fact that they can form polyhedron faces whose normals have fixed directions. For take any point of reference $O$, and take planes $\mathrm{P}_{1}, \mathrm{P}_{2}, \mathrm{P}_{3} \ldots$ through $\mathrm{O}$, parallel to $\sigma_{1}, \sigma_{2}, \sigma_{3} \ldots$.

Cousider any point $A$; let the perpendiculars from $O$ on the surfaces of the polyhedron be $n_{1}, n_{2}, n_{3} \ldots$, and from $\mathrm{A}$ be $n_{1}{ }^{a}, n_{2}{ }^{a}, n_{3}{ }^{a} \ldots$; and let the perpendiculars from $A$ on $\mathrm{P}_{1}, \mathrm{P}_{2}, \mathrm{P}_{3} \ldots$ be $a_{1}, a_{2}, a_{3}, a_{4}, a_{5}, \ldots$ (considered positive or negative, as $\mathrm{A}$ and the corresponding polyhedron surface are on the same or different sides of the corresponding plane $P$ ). Then $\Sigma n s=\Sigma n^{a} s$; for each $=3 \mathrm{~V}$; and

$$
\therefore \Sigma\left(n-u^{a}\right) s=0 \text {, and } \therefore \Sigma a s=0 \text {, (for } n_{1}-u_{1}{ }^{n}=a_{1} \text {, \&c.). }
$$

Now $a_{1}, a_{2}, a_{3} \ldots$ are sufficient to completely fix the position of the point $A$ with reference to 0 , and there are linear relations of the type

$$
a_{4}=p_{4} a_{1}+q_{4} a_{2}+r_{4} a_{3} ; \quad a_{5}=p_{5} a_{1}+y_{5} a_{2}+r_{5} a_{3} ; \quad \& c .
$$

Take other points $\mathrm{B}, \mathrm{C}, \mathrm{D}, \ldots$ and let $b, c, d, \ldots$ correspond to a. Then we bave $\Sigma a s=0, \Sigma l s=0, \Sigma e s=0$, $\Sigma d s=0, \ldots ;$ an indefinite number of relations, but only equivalent to 3 independent relations; for choosing $\lambda, \mu, \nu$ so that

$\lambda a_{1}+\mu b_{1}+\nu c_{1}=d_{1} ; \quad \lambda a_{2}+\mu b_{2}+v c_{2}=d_{2} ; \lambda a_{3}+\mu b_{3}+\nu c_{3}=d_{3} ;$ we have

$$
\begin{aligned}
d_{4} & =p_{4} d_{1}+g_{4} d_{2}+r_{4} d_{3}=p_{4}\left(\lambda a_{1}+\mu b_{1}+\nu c_{1}\right)+q_{4}\left(\lambda a_{2}+\mu l_{2}+\nu c_{2}\right) \\
& +r_{4}\left(\lambda a_{3}+\mu b_{3}+\nu c_{3}\right)=\lambda\left(p_{4} a_{1}+q_{4} a_{2}+r_{4} a_{3}\right)+\mu\left(p_{4} b_{1}+q_{4} l_{2}+r_{4} l_{3}\right) \\
& +\nu\left(p_{4} c_{1}+g_{4} c_{2}+r_{4} c_{3}\right)=\lambda a_{4}+\mu b_{4}+\nu c_{4} ;
\end{aligned}
$$

and similarly

and hence

$$
d_{5}=\lambda a_{5}+\mu b_{5}+\nu c_{5}, \& \mathrm{c} \text {; }
$$

We have then

$$
\Sigma d s-\lambda \Sigma a s+\mu \Sigma(b s+\nu \Sigma c s .
$$

$$
\Sigma n d s=2 d \mathrm{~V} ; \quad \Sigma k d s=d \mathrm{l} ; \text { and } \Sigma a d s=\Sigma b d s=\Sigma c d s=0 ;
$$


and for $\mathrm{V}$ constant and $\mathrm{E}$ a minimum we have

$$
0=\Sigma n d s=\Sigma k d s=\Sigma a d s=\Sigma b d s=\Sigma c d s ;
$$

where the quantities $d s$ are connected only by these five equations.

Hence we have

$$
\begin{gathered}
n_{1}-x a_{1}-\beta b_{1}-\gamma c_{1}-\rho k_{1}=0, \\
n_{2}-\alpha a_{2}-\beta b_{2}-\gamma c_{2}-\rho k_{2}=0, \\
n_{3}-\alpha a_{3}-\beta b_{3}-\gamma c_{3}-\rho k_{3}=0, \\
\& c . \quad \& c .
\end{gathered}
$$

where $\alpha, \beta, \gamma, \rho$ are (unknown) constants.

Now there ean always be found a point $\mathrm{O}_{1}$, whose distances from $\mathrm{P}_{1}, \mathrm{P}_{2}, \mathrm{P}_{3}$ are

$$
\alpha a_{1}+\beta b_{1}+\gamma c_{1}, \quad \alpha a_{2}+\beta b_{2}+\gamma c_{2}, \quad \alpha a_{3}+\beta b_{3}+\gamma c_{3}
$$

respectively; the distance of $\mathrm{O}_{1}$ from $\mathrm{P}_{4}$ is

$$
\begin{aligned}
& p_{4}\left(\alpha a_{1}+\beta b_{1}+\gamma c_{1}\right)+q_{4}\left(\alpha a_{2}+\beta b_{2}+\gamma c_{2}\right)+r_{4}\left(\alpha a_{3}+\beta b_{3}+\gamma c_{3}\right) \\
& =\alpha\left(p_{4} a_{1}+q_{4} a_{2}+r_{4} \alpha_{3}\right)+\beta\left(p_{4} b_{1}+q_{4} l_{2}+r_{4} b_{3}\right) \\
& +\gamma\left(p_{4} c_{1}+q_{4} c_{2}+r_{4} c_{3}\right)=\alpha a_{4}+\beta b_{4}+\gamma c_{4} .
\end{aligned}
$$

Similarly its distance from $\mathrm{P}_{5}$ is $\alpha a_{5}+\beta b_{5}+\gamma c_{\tilde{j}}$, \&c.

Hence if the perpendiculars from $O_{1}$ on $\sigma_{1}, \sigma_{2}, \sigma_{3}, \ldots$ are $n_{2}{ }^{\prime}, n_{2}{ }^{\prime}, n_{3}{ }^{\prime} \ldots$, we have

$$
\begin{gathered}
n_{1}^{\prime}=n_{1}-\alpha t_{1}-\beta b_{1}-\gamma c_{1}=\rho k_{1}, \quad n_{2}^{\prime}=n_{2}-\alpha a_{2}-\beta l_{1}-\gamma c_{2}=\rho k_{2}, \\
n_{3}^{\prime}=n_{3}-\alpha a_{3}-\beta b_{3}-\gamma c_{3}=\rho k_{3}, \text { \&c. \&c. }
\end{gathered}
$$

Hence there is a point $O_{1}$ for which

$$
n_{1}^{\prime}: n_{2}{ }^{\prime}: n_{3}{ }^{\prime} \ldots=k_{1}: l_{2}: k_{3} \ldots,
$$

which is Wulff's theorem.

It is not always easy to apply the above theorem to the case of an actual crystal. It seems therefore worth while to give an example which is of use in many cases.

Let three planes $\mathrm{OBC}, \mathrm{OCA}, \mathrm{OAB}$ meet along the lines $\mathrm{OA}, \mathrm{OB}, \mathrm{OC}$; and let them be met by two planes $\mathrm{ABC}$, $\mathrm{A}^{\prime} \mathrm{B}^{\prime} \mathrm{C}^{\prime}$, so that $\mathrm{OA}=x a, \quad \mathrm{OB}=x b, \quad \mathrm{OC}=x c ; \quad \mathrm{OA}^{\prime}=y a^{\prime}$, $\mathrm{OB}^{\prime}=y b^{\prime}, \mathrm{OC}^{\prime}=y c^{\prime}$, where $a, b, c, a^{\prime}, b^{\prime}, e^{\prime}$ are supposed known. Let the angles $\mathrm{BOC}, \mathrm{COA}, \mathrm{AOB}$ be $\alpha, \beta, \gamma$, respectively.

Then the volume of the figure $O A B C$ is

$$
\frac{x^{3} a b c}{6} \sqrt{1+2 \cos \alpha \cdot \cos \beta \cdot \cos \gamma-\cos ^{2} \alpha-\cos ^{2} \beta-\cos ^{2} \gamma,}
$$

and of $O \mathrm{~A}^{\prime} \mathrm{B}^{\prime} \mathrm{C}^{\prime}$ is

$$
\frac{y^{3} a^{\prime} b^{\prime} c^{\prime}}{6} v^{\prime} 1+2 \cos \alpha \cdot \cos \beta \cdot \cos \gamma-\cos ^{2} \alpha-\cos ^{2} \beta-\cos ^{2} \gamma ;
$$


the area of $A B C$ is

$$
\frac{x^{2}}{2} \sqrt{\mathbf{\Sigma}\left(b^{2} e^{2}\right.} \sin ^{2} \alpha-b c\left(b^{2}+e^{2}\right) \overline{\left.\cos \alpha+2 a^{2} b c \cos \beta \cdot \cos \gamma\right)} \text {; }
$$

the area of $\mathrm{OBC}$ is $\frac{x^{2}}{2} b c \sin \alpha$, and of $\mathrm{OB}^{\prime} \mathrm{C}^{\prime}$ is $\frac{y^{2} b^{\prime} c^{\prime}}{2}-\sin \alpha$; and we get the areas of $A^{\prime} B^{\prime} C^{\prime}, O C A, O C^{\prime} A^{\prime}, O A B, O A^{\prime} B^{\prime}$ similarly.

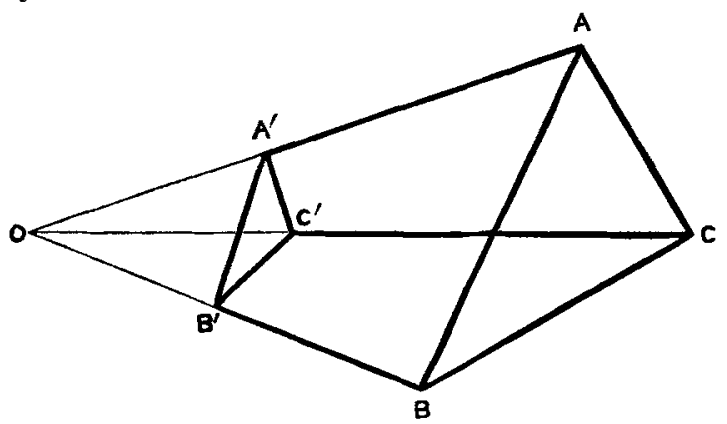

Suppose now that the figure $\mathrm{ABCA}^{\prime} \mathrm{B}^{\prime} \mathrm{C}^{\prime}$ represents a crystal which is such that its surface energy is a minimum for a given volume. Let the capillarity constants of the faces $\mathrm{B}\left(\mathrm{B}^{\prime} \mathrm{C}^{\prime}, \mathrm{CAC}^{\prime} \mathrm{A}^{\prime}, \mathrm{ABA}^{\prime} \mathrm{B}^{\prime}, \mathrm{AB}(), \mathrm{A}^{\prime} \mathrm{B}^{\prime} \mathrm{C}^{\prime}\right.$ be $k_{1}, k_{2}, k_{3}, k$, and $k^{\prime}$, respectively; then the volume of the crystal is

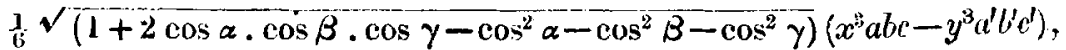
and the surface energy is $P_{1} x^{2}-P_{2} y^{2}$, where $\mathrm{P}_{1}=\frac{1}{2}\left(b c k_{1} \sin \alpha+c a k_{2} \sin \beta+a b k_{3} \sin \gamma\right.$

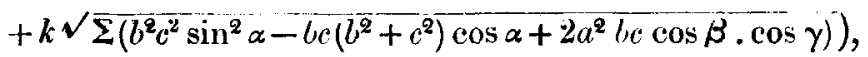
$P_{2}=\frac{1}{=}\left(b^{\prime} c^{\prime} k_{1} \sin a+c^{\prime} d^{\prime} k_{2} \sin \beta+a^{\prime} l^{\prime} k_{3} \sin \gamma\right.$ $\left.-k^{\prime} \sqrt{\sum\left(b^{12} c^{12} \sin ^{2} \alpha-b^{\prime} c^{\prime}\left(b^{12}+c^{\prime 2}\right) \cos \alpha+2 a^{12} b^{\prime} c^{\prime} \cos \beta \cdot \cos \gamma\right)}\right)$.

We have then at once for constant volume and surface energy a minimum, on differentiating

$$
y_{y}^{x}=\frac{\mathrm{P}_{1} a^{\prime} b^{\prime} f^{\prime}}{\mathrm{P}_{2} a l y} \text {; with the condition } \frac{\mathrm{P}_{1}^{4} a^{2} b^{\prime 2} e^{\prime 2}}{\mathrm{P}_{2}^{3} a^{2} b^{2} c^{2}}-\mathrm{P}_{1}>0 \text {. }
$$

Magdalen College, (Oxford. 